\title{
Dual Numbers Approach in Multiaxis Machines Error Modeling
}

\author{
Jaroslav Hrdina and Petr Vašík \\ Institute of Mathematics, Faculty of Mechanical Engineering, Brno University of Technology, 61669 Brno, Czech Republic \\ Correspondence should be addressed to Jaroslav Hrdina; hrdina@fme.vutbr.cz
}

Received 21 March 2014; Revised 19 May 2014; Accepted 21 May 2014; Published 1 June 2014

Academic Editor: Shi-Liang Wu

Copyright (C) 2014 J. Hrdina and P. Vašík. This is an open access article distributed under the Creative Commons Attribution License, which permits unrestricted use, distribution, and reproduction in any medium, provided the original work is properly cited.

Multiaxis machines error modeling is set in the context of modern differential geometry and linear algebra. We apply special classes of matrices over dual numbers and propose a generalization of such concept by means of general Weil algebras. We show that the classification of the geometric errors follows directly from the algebraic properties of the matrices over dual numbers and thus the calculus over the dual numbers is the proper tool for the methodology of multiaxis machines error modeling.

\section{Introduction}

The concepts of multiaxis machines error modeling can be found in classical literature $[1,2]$, including the appropriate methodology. For the process description, the homogeneous transformation matrices (HTM) are used as the crucial mathematical tool for the error models; see $[3,4]$.

The complex multiaxis machine positioning is represented by a kinematic chain. Thus, by means of the product of the transformations between successive coordinate systems associated to the mechanisms elements, from the absolute reference system to the tool reference system, the global transformation matrix $T \in \mathrm{Mat}_{4}(\mathbb{R})$ is obtained. The basic setting takes place in the affine extension of vector space $\mathbb{R}^{3}$. This approach appears quite often in modern literature with minor modifications; see [5-8]; for a rare attempt to use modern advanced mathematical structures such as the algebra of quaternions, see [9].

The main uncertainty sources in the design and construction of machine tools are geometric and kinematic errors, thermal errors, stiffness error, and errors addressed to the deflection of cutting tools. Those mentioned above are the known sources. Their consequences are complex, but techniques to evaluate them or compensate their effects have being developed; see [10, 11]. In our paper we work with geometric and kinematic errors in any machine component which can be considered in the kinematic model as a new parameter.
For example, the coordinate transformation matrices and the corresponding error matrices for three-axis machines are

$$
\begin{aligned}
T_{1} & =\left(\begin{array}{llll}
1 & 0 & 0 & x \\
0 & 1 & 0 & 0 \\
0 & 0 & 1 & 0 \\
0 & 0 & 0 & 1
\end{array}\right), \\
E_{1} & =\left(\begin{array}{cccc}
1 & -\epsilon_{z x} & \epsilon_{y x} & \delta_{x x} \\
\epsilon_{z x} & 1 & -\epsilon_{x x} & \delta_{y x} \\
-\epsilon_{y x} & \epsilon_{x x} & 1 & \delta_{z x} \\
0 & 0 & 0 & 1
\end{array}\right), \\
T_{2} & =\left(\begin{array}{cccc}
1 & 0 & 0 & 0 \\
0 & 1 & 0 & y \\
0 & 0 & 1 & 0 \\
0 & 0 & 0 & 1
\end{array}\right), \\
E_{2} & =\left(\begin{array}{cccc}
1 & -\epsilon_{z y} & \epsilon_{y y} & \delta_{x y}-S_{x y} y \\
\epsilon_{z y} & 1 & -\epsilon_{x y} & \delta_{y y} \\
-\epsilon_{y y} & \epsilon_{x y} & 1 & \delta_{z y} \\
0 & 0 & 0 & 1
\end{array}\right), \\
T_{3} & =\left(\begin{array}{cccc}
1 & 0 & 0 & 0 \\
0 & 1 & 0 & 0 \\
0 & 0 & 1 & z \\
0 & 0 & 0 & 1
\end{array}\right), \\
E_{3} & =\left(\begin{array}{cccc}
1 & -\epsilon_{z z} & \epsilon_{y z} & \delta_{x z}-S_{x z} z \\
\epsilon_{z z} & 1 & -\epsilon_{x z} & \delta_{y z}-S_{y z} z \\
-\epsilon_{y z} & \epsilon_{x z} \\
0 & 0 & 0 & \delta_{z z} \\
0 & 0 & 1
\end{array}\right),
\end{aligned}
$$


where $\delta_{i i}$ denotes the linear errors along the $i$ th axis, $\delta_{i j}, i \neq j$ is the straightness errors in the $i$ th axis direction when moving along the $j$ th axis, $\epsilon_{i j}$ is the angular errors around the $i$ th axis when moving along the $j$ th axis, and $S_{i j}$ is the squareness errors between the corresponding axes.

Our goal is to set the methodology of multiaxis machines geometric error in the context of modern theory of Weil algebras [12]. In this paper, we essentially use Weil algebra $\mathbb{D}_{1}^{1}$ and in the final section we present the directions of further research and discuss the advantages of employing more general Weil algebras into the error analysis. When assembling the kinematic chain containing geometric errors, we embed the error matrix corresponding to any kinematic joint, that is, the errors of joint translation or rotation. In particular, for the translation in the vector $(x, y, z)$ direction or for the rotation around the $z$ axis by the angle $\gamma$, the following error matrices apply, respectively:

$$
\left(\begin{array}{cccc}
1 & -\alpha & \beta & x \\
\alpha & 1 & \gamma & y \\
-\beta & -\gamma & 1 & z \\
0 & 0 & 0 & 1
\end{array}\right)\left(\begin{array}{cccc}
1 & -\alpha \cos (\theta+\gamma)-\beta \sin (\theta+\gamma) & \alpha \sin (\theta+\gamma)-\beta \cos (\theta+\gamma) & 0 \\
\alpha & \cos (\theta+\gamma) & -\sin (\theta+\gamma) & 0 \\
-\beta & \sin (\theta+\gamma) & \cos (\theta+\gamma) & 0 \\
0 & 0 & 0 & 1
\end{array}\right)
$$

The parameters $\alpha, \beta$, and $\gamma$ represent the error rotations around the axes $z, y$, and $x$, respectively, and $\theta$ gives the proper rotation around axis $z$. The error matrices were derived from the rotation matrices around particular axes by approximation. More precisely, for the translation of the rotation around axis $x$, error matrix is approximated as follows:

$$
\left(\begin{array}{cccc}
1 & 0 & 0 & 0 \\
0 & \cos \alpha & -\sin \alpha & 0 \\
0 & \sin \alpha & \cos \alpha & 0 \\
0 & 0 & 0 & 1
\end{array}\right) \leadsto\left(\begin{array}{cccc}
1 & 0 & 0 & 0 \\
0 & 1 & -\alpha & 0 \\
0 & \alpha & 1 & 0 \\
0 & 0 & 0 & 1
\end{array}\right)
$$

Thus $\cos x \rightsquigarrow 1$ and $\sin x \rightsquigarrow x$. In case two approximations are multiplied, the whole term vanishes. This is caused by the assumption that the errors are by order smaller than the proper rotation parameters. The above mentioned representation is a standard description of the error matrices to be embedded into the kinematic chain.

For example, in case of two-axis machines with two translation joints, we obtain the following kinematic chain:

$$
\begin{aligned}
& \left(\begin{array}{cccc}
1 & -\alpha_{1} & \beta_{1} & x_{1} \\
\alpha_{1} & 1 & \gamma_{1} & y_{1} \\
-\beta_{1} & -\gamma_{1} & 1 & z_{1} \\
0 & 0 & 0 & 1
\end{array}\right)\left(\begin{array}{llll}
1 & 0 & 0 & \bar{x} \\
0 & 1 & 0 & 0 \\
0 & 0 & 1 & 0 \\
0 & 0 & 0 & 1
\end{array}\right) \\
& \times\left(\begin{array}{cccc}
1 & -\alpha_{2} & \beta_{2} & x_{2} \\
\alpha_{2} & 1 & \gamma_{2} & y_{2} \\
-\beta_{2} & -\gamma_{2} & 1 & z_{2} \\
0 & 0 & 0 & 1
\end{array}\right)\left(\begin{array}{llll}
1 & 0 & 0 & 0 \\
0 & 1 & 0 & \bar{y} \\
0 & 0 & 1 & 0 \\
0 & 0 & 0 & 1
\end{array}\right) .
\end{aligned}
$$

If the above mentioned identities $\alpha_{i} \beta_{j}=0, \alpha_{i} \gamma_{j}=0$, and $\beta_{i} \gamma_{j}=0$ for all $i, j \in\{1,2\}$ are applied, we obtain the matix

$$
\begin{gathered}
n\left(\begin{array}{cccc}
1 & -\alpha_{1} & \beta_{1} & \bar{x}+x_{1} \\
\alpha_{1} & 1 & \gamma_{1} & \alpha_{1} \bar{x}+y_{1} \\
-\beta_{1} & -\gamma_{1} & 1 & -\beta \bar{x}+z_{1} \\
0 & 0 & 0 & 1
\end{array}\right)\left(\begin{array}{cccc}
1 & -\alpha_{2} & \beta_{2} & -\alpha_{2} \bar{y}+x_{2} \\
\alpha_{2} & 1 & \gamma_{2} & \bar{y}+y_{2} \\
-\beta_{2} & -\gamma_{2} & 1 & \gamma_{2} \bar{y}+z_{2} \\
0 & 0 & 0 & 1
\end{array}\right) \\
\quad=\left(\begin{array}{cccc}
1 & -\alpha_{1}-\alpha_{2} & \beta_{1}+\beta_{2} & -\alpha_{2} \bar{y}+\bar{x}+x_{1} \\
\alpha_{1}+\alpha_{2} & 1 & \gamma_{1}+\gamma_{2} & \bar{y}+y_{2}+\alpha_{1} \bar{x}+y_{1} \\
-\beta_{2}-\beta_{2} & -\gamma_{1}-\gamma_{2} & 1 & \gamma_{2} \bar{y}+z_{2}-\beta_{1} \bar{x}+z_{1} \\
0 & 0 & 0 & 1
\end{array}\right)
\end{gathered}
$$

and the corresponding kinematic equations which are to be solved within the error analysis. Generally, in case of the system of linear equations, we proceed by Gauss elimination; for nonlinear systems we use Gröbner bases. In our setting, we compute with the matrices using the identities $\alpha_{i} \beta_{j}=0$, $\alpha_{i} \gamma_{j}=0$ and $\beta_{i} \gamma_{j}=0$ for all $i, j \in\{1,2\}$, which resemble the identities for the imaginary parts of the dual numbers. Thus it makes sense for the whole theory to use the homogeneous transformation matrices over the dual numbers. Our further approach to error calculations will thus be based on the dual numbers calculus; more generally we use a Weil algebra. This gives us a formal setting for the geometric errors modeling.

\section{Matrices over Dual Numbers}

As we are going to calculate with matrices over a structure different from real or complex numbers, moreover a structure which is not a field but a ring only, we have to guarantee that the calculations within the inverse kinematics make sense. In mathematical language, we need the dual numbers to form the so-called Euclidean domain.

By an Euclidean domain $R$ we understand an integral domain which is endowed with at least one Euclidean function, that is, function of the form $f: R-\left\{0_{R}\right\} \rightarrow \mathbb{Z}_{0}^{+}$satisfying the following property: if $a, b \in R$ and $b \neq 0_{R}$, then there are $q$ and $r \in R$ such that $a=b q+r$ and either $r=0_{R}$ or $f(r)<f(b)$.

Let us recall that for any field we shall define $f(x)=1$ for any nonzero $x$ and thus any field is Euclidean. The most important property of the Euclidean domain is that the Euclidean algorithm can be used to find the greatest common divisor of its two elements (i.e., one can easily see that any Euclidean domain is a principal ideal domain-PID). This leads to the fact that in Euclidean domains the Gauss elimination method for solving systems of linear equations can be applied.

As an example of Euclidean domain, let us recall three well-known extensions of real numbers. 
Consider

$$
\begin{aligned}
& \mathbb{C}=\left\{a+\text { bia }, b \in \mathbb{R}, i^{2}=-1\right\}, \\
& \mathbb{D}=\left\{a+\text { bia }, b \in \mathbb{R}, i^{2}=0\right\}, \\
& \mathbb{P}=\left\{a+\text { bia }, b \in \mathbb{R}, i^{2}=1\right\} .
\end{aligned}
$$

Rings $\mathbb{C}, \mathbb{D}$, and $\mathbb{P}$ can be obtained as factor rings of $\mathbb{R}[x]$ : $\mathbb{C}=\mathbb{R}[x] /\left(x^{2}+1\right), \mathbb{D}=\mathbb{R}[x] /\left(x^{2}\right)$, and $\mathbb{P}=R[x] /\left(x^{2}-1\right)$. Only $x^{2}+1$ is irreducible over $\mathbb{R}$; thereby $\mathbb{C}$ is a field. Neither $\mathbb{D}$ nor $\mathbb{P}$ are integral domains: we have $a i \times b i=0$ for $\mathbb{D}$ and $(a+a i) \times(b-b i)=0$ for $\mathbb{P}$ (by equations also presented all zero divisors of these rings).

In particular, the dual numbers extend the real numbers by adjoining one new element $\epsilon$ with the property $\epsilon^{2}=0(\epsilon$ is nilpotent). The collection of dual numbers forms a particular two-dimensional commutative unital associative algebra over the real numbers. Every dual number has the form

$$
z=a+b \epsilon
$$

with $a$ and $b$ uniquely determined real numbers. Division of dual numbers is defined when the real part of the denominator is nonzero. The division process is analogous to complex division in that the denominator is multiplied by its conjugate in order to cancel the nonreal parts.

We define the following class of matrices over dual numbers:

$$
\text { Mat }_{n} \mathbb{D}=\left\{A+\epsilon B \mid A, B \in \text { Mat }_{n} \mathbb{R}\right\}
$$

and the group of orthogonal matrices over dual numbers:

$$
O(n, \mathbb{D})=\left\{C \in \mathrm{Mat}_{n} \mathbb{D} \mid C^{T} C=E\right\} .
$$

Indeed, the set $O(n, \mathbb{D})$ with standard matrix multiplication is a group as it is closed under the operation, more precisely $(A B)^{T} A B=B^{T} A^{T} A B=B^{T} B=E$; the inverse to the matrix $A$ is the matrix $A^{T}$ and the unitary matrix is of the form $E+\epsilon 0$.

Theorem 1. An element $A+\epsilon B \in$ Mat $_{n} \mathbb{D}$ lies in $O(n, \mathbb{D})$ if $A \in O(n)=O(n, \mathbb{R})$ and $B$ satisfies the identity

$$
A^{T} B=-B^{T} A \text {. }
$$

Proof. $(A+\epsilon B)^{T}(A+\epsilon B)=\left(A^{T}+\epsilon B^{T}\right)(A+\epsilon B)=A^{T} A+$ $\epsilon\left(A^{T} B+B^{T} A\right)$ and thus $A^{T} A=E$ a $A^{T} B+B^{T} A$.

Definition 2. Let $A \in O(n)$. Then the set

$$
O^{A}=\left\{A+B \epsilon \mid A^{T} B+A B^{T}=0\right\}
$$

is called the dual extension of the matrix $A$ and the set

$$
I^{A}=\left\{B \mid A^{T} B+A B^{T}=0\right\}
$$

of the imaginary parts of the matrices within the class $O^{A}$ is called the $A$-admissible error class or shortly the error class.

Let us note that in the special case $A=E$, the error class $I^{E}$ is a Lie algebra $\mathfrak{g o}(n)$.
Lemma 3. The error class $I^{A}$ is a Lie algebra with respect to the commutator operation $[F, G]=F G-G F$ if and only if $A$ is symmetric, that is, $A^{T}=A$.

Proof. For $A \in O(n), B, C \in I^{A}$ holds the following: $A^{T}[B, C]=$ $A^{T}(B C-C B)=A^{T} B C-A^{T} C B=-B^{T} A C+C^{T} A B=$ $B^{T} C^{T} A^{T}-C^{T} B^{T} A^{T}=\left((C B)^{T}-(B C)^{T}\right) A^{T}=(C B-B C)^{T} A^{T}=$ $[C, B]^{T} A^{T}=-[B, C]^{T} A^{T}$.

Example. Another possible choice of the matrix $A$ such that $I^{A}$ is an algebra is $A=\bar{E}$, where

$$
\bar{E}=\left(\begin{array}{cc}
0 & E \\
E & 0
\end{array}\right) \in \mathrm{Mat}_{2 n} \mathbb{R},
$$

where $E$ is an identity $n \times n$ matrix. Indeed, for another matrix $\left(\begin{array}{ll}M & N \\ O & P\end{array}\right) \in I^{\bar{E}}$ with $M, N, O, P \in \mathrm{Mat}_{n} \mathbb{R}$ we obtain

$$
\left(\begin{array}{ll}
0 & E \\
E & 0
\end{array}\right)\left(\begin{array}{cc}
M & N \\
O & P
\end{array}\right)=-\left(\begin{array}{cc}
M & O \\
N & P
\end{array}\right)\left(\begin{array}{cc}
0 & E \\
E & 0
\end{array}\right)
$$

and thus

$$
I^{\bar{E}}=\left(\begin{array}{cc}
M & 0 \\
0 & -M
\end{array}\right) \cong \operatorname{Mat}_{n} \mathbb{R}
$$

Furthermore, the set

$$
S O(n, \mathbb{D})=\{A+\epsilon B \in O(n, \mathbb{D}) \mid A \in S O(n)\}
$$

will be called the class of the special orthogonal matrices over $\mathbb{D}$.

Lemma 4. Let $A+B \epsilon \in S O(2, \mathbb{D})$. Then $A+B \epsilon$ is of the form

$$
\left(\begin{array}{cc}
\cos (\varphi)-k \operatorname{tg}(\varphi) \epsilon & -\sin (\varphi)-k \epsilon \\
\sin (\varphi)+k \epsilon & \cos (\varphi)-k \operatorname{tg}(\varphi) \epsilon
\end{array}\right)
$$

where $\varphi \in\langle 0,2 \pi\rangle$, and $k \in \mathbb{R}$.

Proof. Because $A \in S O(2)$, it has to be of the form

$$
A=\left(\begin{array}{cc}
\cos (\varphi) & -\sin (\varphi) \\
\sin (\varphi) & \cos (\varphi)
\end{array}\right)
$$

Furthermore, let us consider a matrix $B$ in the general form $\left(\begin{array}{ll}a & b \\ c & d\end{array}\right)$. As $A+B \epsilon \in S O(2, \mathbb{D})$, the identity (10) has to be fulfilled:

$$
\begin{aligned}
& \left(\begin{array}{cc}
\cos (\varphi) & \sin (\varphi) \\
-\sin (\varphi) & \cos (\varphi)
\end{array}\right)\left(\begin{array}{ll}
a & b \\
c & d
\end{array}\right) \\
& =-\left(\begin{array}{ll}
a & c \\
b & d
\end{array}\right)\left(\begin{array}{cc}
\cos (\varphi) & -\sin (\varphi) \\
\sin (\varphi) & \cos (\varphi)
\end{array}\right)
\end{aligned}
$$

This leads to the following equations:

$$
\begin{gathered}
a \cos (\varphi)+c \sin (\varphi)=-a \cos (\varphi)-c \sin (\varphi), \\
b \cos (\varphi)+d \sin (\varphi)=a \sin (\varphi)-c \cos (\varphi), \\
-a \sin (\varphi)+c \cos (\varphi)=-b \cos (\varphi)-d \sin (\varphi), \\
-b \sin (\varphi)+d \cos (\varphi)=b \sin (\varphi)-d \cos (\varphi) .
\end{gathered}
$$


From the first and the last one we obtain identities $a=$ $-\operatorname{ctg}(\varphi)$ and $d=\operatorname{btg}(\varphi)$. If we substitute these into the rest of the equalities, we obtain the following calculations:

$$
\begin{aligned}
b \cos (\varphi)+b \operatorname{tg}(\varphi) \sin (\varphi) & =-c t g(\varphi) \sin (\varphi)-c \cos (\varphi) \\
b(\cos (\varphi))^{2}+b\left(\sin (\varphi)^{2}\right) & =-c(\sin (\varphi))^{2}-c(\cos (\varphi))^{2}, \\
b & =-c .
\end{aligned}
$$

Thus for the matrix $A$ we conclude that

$$
I^{A}=\left(\begin{array}{cc}
-k \operatorname{tg}(\varphi) \epsilon & -k \epsilon \\
k \epsilon & -k \operatorname{tg}(\varphi) \epsilon
\end{array}\right), \quad k \in \mathbb{R},
$$

which completes the proof.

Lemma 5. Let $A, B \in O(n)$ then

$$
\left(A+I^{A} \epsilon\right)\left(B+I^{B} \epsilon\right)=A B+I^{A B} \epsilon \text {. }
$$

Proof. $\left(A+I^{A} \epsilon\right)\left(B+I^{B} \epsilon\right)=A B+\left(A I^{B}+I^{A} B\right) \epsilon$ and it is easy to see that $\left(A I^{B}+I^{A} B\right) \in I^{A B}$ :

$$
\begin{aligned}
(A B)^{T} & \left(A I^{B}+I^{A} B\right)+\left(A I^{B}+I^{A} B\right)^{T} A B \\
& =B^{T} A^{T}\left(A I^{B}+I^{A} B\right)+\left(\left(I^{B}\right)^{T} A^{T}+B^{T}\left(I^{A}\right)^{T}\right) A B \\
& =B^{T} I^{B}+B^{T} A^{T} I^{A} B+\left(I^{B}\right)^{T} B+B^{T}\left(I^{A}\right)^{T} A B \\
& =B^{T} I^{B}+\left(I^{B}\right)^{T} B+B^{T}\left(A^{T} I^{A}+\left(I^{A}\right)^{T} A\right) B=0 .
\end{aligned}
$$

On the other hand, if $C \in I^{A B}$ then one can find $C_{1} \in I^{B}$ and $C_{2} \in I^{A}$ such that $A C_{1}+C_{2} B=C$; for any $C_{2} \in I^{A}$ we can define $C_{1}=A^{T} C-A^{T} C_{2} B$, such that $C_{1} \in I^{B}$ :

$$
\begin{aligned}
& B^{T}\left(A^{T} C-A^{T} C_{2} B\right)+\left(A^{T} C-A^{T} C_{2} B\right)^{T} B \\
& \quad=B^{T} A^{T} C-B^{T} A^{T} C_{2} B+\left(C^{T} A-B^{T} C_{2}^{T} A\right) B \\
& =B^{T} A^{T} C-B^{T} A^{T} C_{2} B+C^{T} A B-B^{T} C_{2}^{T} A B=0 .
\end{aligned}
$$

Theorem 6. Let $A+B \epsilon \in S O(3, \mathbb{D})$. Then $A+B \epsilon$ is of the form

$$
R_{1} R_{2} R_{3}+\left(R_{1} R_{2} I^{R_{3}}+R_{1} I^{R_{2}} R_{3}+I^{R_{1}} R_{2} R_{3}\right) \epsilon,
$$

where $R_{1}, R_{2}$, and $R_{3}$ are the matrices of rotations around the axes $x, y$, and $z$, respectively, and

$$
\begin{aligned}
& I^{R_{1}}=\left(\begin{array}{ccc}
0 & -\alpha \cos (\varphi)-\beta \sin (\varphi) & \alpha \sin (\varphi)-\beta \cos (\varphi) \\
\alpha & -k \operatorname{tg}(\varphi) & -k \\
\beta & k & -k \operatorname{tg}(\varphi)
\end{array}\right) \text {, } \\
& I^{R_{2}}=\left(\begin{array}{ccc}
-k \operatorname{tg}(\varphi) & \alpha & -k \\
-\alpha \cos (\varphi)-\beta \sin (\varphi) & 0 & \alpha \sin (\varphi)-\beta \cos (\varphi) \\
k & \beta & -k \operatorname{tg}(\varphi)
\end{array}\right), \\
& I^{R_{3}}=\left(\begin{array}{ccc}
-k \operatorname{tg}(\varphi) & -k & \alpha \\
k & -k \operatorname{tg}(\varphi) & \beta \\
-\alpha \cos (\varphi)-\beta \sin (\varphi) & \alpha \sin (\varphi)-\beta \cos (\varphi) & 0
\end{array}\right) .
\end{aligned}
$$

Proof. It is known that any element $A \in S O(3)$ can be represented as a composition of three rotation matrices $R_{1}, R_{2}, R_{3}$ (around axes $x, y$, and $z$, resp.). Thus if the extension to $\mathrm{SO}(3, \mathbb{D})$ is considered, we obtain

$$
\begin{aligned}
& \left(R_{1}+I^{R_{1}} \epsilon\right)\left(R_{2}+I^{R_{2}} \epsilon\right)\left(R_{3}+I^{R_{3}} \epsilon\right) \\
& \quad=R_{1} R_{2} R_{3}+\left(R_{1} R_{2} I^{R_{3}}+R_{1} I^{R_{2}} R_{3}+I^{R_{1}} R_{2} R_{3}\right) \epsilon
\end{aligned}
$$

and $A=R_{1} R_{2} R_{3} \in S O(3)$, according to Lemma 5 .

Let us now consider the rotation around the axis $x$ in particular. The matrix $A$ will be of the form $\left(\begin{array}{ll}1 & 0 \\ 0 & M\end{array}\right)$, where $M \in$ $S O(2)$. If we consider a general error matrix of such type in the form $\left(\begin{array}{ll}a & x \\ y & N\end{array}\right)$, where $a \in \mathbb{R}$ and $x, y$ are the dimension two row and column vectors, respectively, from the identity (10) we obtain

$$
\begin{aligned}
\left(\begin{array}{cc}
1 & 0 \\
0 & M^{T}
\end{array}\right)\left(\begin{array}{ll}
a & x \\
y & N
\end{array}\right) & =-\left(\begin{array}{cc}
a & y^{T} \\
x^{T} & N^{T}
\end{array}\right)\left(\begin{array}{ll}
1 & 0 \\
0 & M
\end{array}\right), \\
\left(\begin{array}{cc}
a & x \\
M^{T} y & M^{T} N
\end{array}\right) & =-\left(\begin{array}{cc}
a & y^{T} M \\
x^{T} & N^{T} M
\end{array}\right) .
\end{aligned}
$$

Thus $a=0$ and $x=-y^{T} M$ which is equal to $M^{T} y=x^{T}$ and the identity $M^{T} N=-N^{T} M$ for the dimension two matrices holds from Lemma 4 . If, in addition,

$$
y=\left(\begin{array}{l}
\alpha \\
\beta
\end{array}\right)
$$

then

$$
x=((-\alpha \cos (\varphi)-\beta \sin (\varphi))(\alpha \sin (\varphi)-\beta \cos (\varphi)))
$$

and we obtain that

$$
I^{R_{1}}=\left(\begin{array}{ccc}
0 & -\alpha \cos (\varphi)-\beta \sin (\varphi) & \alpha \sin (\varphi)-\beta \cos (\varphi) \\
\alpha & -k \operatorname{tg}(\varphi) & -k \\
\beta & k & -k \operatorname{tg}(\varphi)
\end{array}\right) .
$$

The remaining rotation represented by $R_{2}$ and $R_{3}$ can be computed similarly. 
Let us note that the matrices $I^{R_{i}}, i \in\{1,2,3\}$ contain the errors $\alpha, \beta$ and if $\varphi$ is understood as the sum $\theta+\gamma$ of the rotation angle $\theta$ and the rotation error angle $\gamma$, then all classical rotation errors are involved and the appropriate error matrix with $k=0$ in addition corresponds to the classical error matrix. The geometric role of the parameter $k$ within the matrix

$$
\left(\begin{array}{ccc}
1 & 0 & 0 \\
0 & -k \operatorname{tg}(\varphi) & -k \\
0 & k & -k \operatorname{tg}(\varphi)
\end{array}\right)
$$

is unknown as it does not appear in the geometric error modeling.

\section{Example}

The following elementary example of two-axis machine will show the methodology of geometric errors modeling. The kinematic chain is described by means of the moving frame method, where the rotation matrices $R_{i} \in S O(n)$ are replaced by the matrices $R_{i}+I^{R_{i}} \in \in S O(n, \mathbb{D})$. It is crucial that $\mathbb{D}$ is the
Euclidean domain and thus the methods of Gauss elimination and Gröbner bases can be used when the inverse kinematics is solved. We will demonstrate the process in the case of twoaxis machine with one rotation axis $x$ and one translation in the direction of axis $y$. Thus, in the following, we shall work in the affine extension of the vector space $\mathbb{R}^{3}$, where vectors $\mathbb{X}=$ $(x, y, z)^{T} \in \mathbb{R}^{3}$ are represented as the elements $(x, y, z, 1)^{T} \in$ $\mathbb{R}^{4}$. The matrix $A \in \operatorname{Mat}_{n}(3, \mathbb{D})$ is then represented by the matrix $\left(\begin{array}{cc}A & \mathbb{X} \\ 0 & 1\end{array}\right) \in \operatorname{Mat}_{n}(3, \mathbb{D}) \rtimes \mathbb{R}^{3} \subset \operatorname{Mat}_{n}(4, \mathbb{D})$. For $T=$ $(A, \mathbb{X}) \in \operatorname{Mat}_{n}(3, \mathbb{D}) \rtimes \mathbb{R}^{3}$ we write $I^{T}:=\left(I^{A}, \mathbb{X}\right)$.

The transformation matrices

$$
T_{1}=\left(\begin{array}{llll}
1 & 0 & 0 & 0 \\
0 & 1 & 0 & y \\
0 & 0 & 1 & 0 \\
0 & 0 & 0 & 1
\end{array}\right), \quad T_{2}=\left(\begin{array}{cccc}
1 & 0 & 0 & 0 \\
0 & \cos (\varphi) & -\sin (\varphi) & 0 \\
0 & \sin (\varphi) & \cos (\varphi) & 0 \\
0 & 0 & 0 & 1
\end{array}\right)
$$

are the elements of $O(3) \rtimes \mathbb{R}^{3}$. When the errors are added, we obtain

$$
\begin{aligned}
T_{1}+I^{T_{1}} \epsilon & =\left(\begin{array}{cccc}
1 & -\alpha_{1} \epsilon & -\beta_{1} \epsilon & x_{1} \epsilon \\
\alpha_{1} \epsilon & 1 & -\gamma_{1} \epsilon & y_{1} \epsilon \\
\beta_{1} \epsilon & \gamma_{1} \epsilon & 1 & z_{1} \epsilon \\
0 & 0 & 0 & 1
\end{array}\right), \\
T_{2}+I^{T 2} & =\left(\begin{array}{cccc}
1+a \epsilon & -\alpha_{2} \cos (\varphi) \epsilon-\beta_{2} \sin (\varphi) \epsilon & \alpha_{2} \sin (\varphi) \epsilon-\beta_{2} \cos (\varphi) \epsilon & x_{2} \epsilon \\
\alpha_{2} \epsilon & \cos (\varphi)-k \operatorname{tg}(\varphi) \epsilon & \sin (\varphi)-k \epsilon & y_{2} \epsilon \\
\beta_{2} \epsilon & \sin (\varphi)+k \epsilon & \cos (\varphi)-k \operatorname{tg}(\varphi) \epsilon & z_{2} \epsilon \\
0 & 0 & 0 & 1
\end{array}\right) .
\end{aligned}
$$

Now the transformation matrices became the elements of $O(3, \mathbb{D}) \rtimes \mathbb{R}^{3}$ (note that it would make sense to consider the calculations in the algebra $O(3, \mathbb{D}) \otimes \mathbb{D}$ instead, but this is not the topic considered in this paper). The resulting matrix is then of the form

$$
\begin{aligned}
& \left(T_{1}+I^{E}\right)\left(T_{2}+I^{T 2}\right) \\
& =\left(\begin{array}{cccc}
a \epsilon & -\cos (\varphi)\left(\alpha_{1}+\alpha_{2}\right) \epsilon-\sin (\varphi)\left(\beta_{1}+\beta_{2}\right) \epsilon & -\sin (\varphi)\left(\alpha_{1}-\alpha_{2}\right) \epsilon-\cos (\varphi)\left(\beta_{1}+\beta_{2}\right) \epsilon & x_{1}+x_{2} \\
\left(\alpha_{1}+\alpha_{2}\right) \epsilon & \cos (\varphi)+\left(-k t g(\varphi)+\gamma_{1} \sin (\varphi)\right) \epsilon & \sin (\varphi)+\left(-k-\gamma_{1} \cos (\varphi)\right) \epsilon & y_{1}+y_{2}+y \\
\left(\beta_{1}+b e_{2}\right) \epsilon & \sin (\varphi)+\left(k+\gamma_{1} \sin (\varphi)\right) \epsilon & \cos (\varphi)+\left(k t g(\varphi)+\gamma_{1} \cos (\varphi)\right) \epsilon & \bar{z}_{1}+z_{2} \\
0 & 0 & 0 & 1
\end{array}\right) .
\end{aligned}
$$

This describes the case of two-axis machine completely. To apply this approach on the three-axis machine, it is enough to extend the kinematic chain by the term $\left(T_{3}+I^{E}\right)$. If, in addition, rotation machine elements are considered, it is necessary to employ the rotation error matrices $\left(R_{i}+I^{R_{i}}\right)$.

\section{Notes on Weil Algebras}

Let $r, k \in \mathbb{N}$ and let us denote $\mathbb{D}_{k}^{r}$ the $\mathbb{R}$-algebra

$$
\mathbb{R} \frac{\left[x_{1}, \ldots, x_{k}\right]}{\left\langle x_{1}, \ldots, x_{n}\right\rangle^{r+1}},
$$


where $\mathbb{R}\left[x_{1}, \ldots, x_{k}\right]$ is the $\mathbb{R}$-algebra of real polynomials in $k$ indeterminates and $\left\langle x_{1}, \ldots, x_{n}\right\rangle^{r+1}$ is the $(r+1)$ th power of its maximal ideals.

Definition 7. Weil algebra is an arbitrary nontrivial quotient $\mathbb{R}$-algebra of $\mathbb{D}_{k}^{r}$. For example, for $\mathbb{D}_{1}^{1}$ we obtain the dual numbers $\mathbb{D}$ and for $\mathbb{D}_{2}^{2}$ we have the set of polynomials in the form $a x+b y+c x^{2}+d x^{2}+e x y$.

Furthermore, the Weil algebra $\mathbb{W}_{1}=\mathbb{D}_{2}^{2} /\langle x y\rangle$ can be defined and the set of polynomials

$$
a x+b y+c x^{2}+d x^{2}
$$

is obtained.

For the sake of the error analysis, the matrix class $R_{1}+I^{R_{1}}$ can be represented by the element of $\mathbb{D}_{4}^{n}$ (where $n$ determines the level of accuracy of the error analysis):

$$
\begin{aligned}
R_{1}+ & \left(\begin{array}{ccc}
a & -\cos (\varphi) & \sin (\varphi) \\
1 & 0 & 0 \\
0 & 0 & 0
\end{array}\right) x_{1} \\
+ & \left(\begin{array}{ccc}
0 & -\sin (\varphi) & -\cos (\varphi) \\
0 & 0 & 0 \\
1 & 0 & 0
\end{array}\right) x_{2}+\left(\begin{array}{lll}
1 & 0 & 0 \\
0 & 0 & 0 \\
0 & 0 & 0
\end{array}\right) x_{3} \\
& +\left(\begin{array}{ccc}
0 & 0 & 0 \\
0 & -\operatorname{tg}(\varphi) & -1 \\
0 & 1 & -\operatorname{tg}(\varphi)
\end{array}\right) x_{4} .
\end{aligned}
$$

The choice $n=1$ neglects the interference of any two errors and the calculations will be similar to those over the dual numbers. In case $n=3$, the actual interference of three errors for the term to be neglected is needed, but with the additional choice of the Weil algebra one can determine those error combinations which can be neglected or eventually replaced.

For instance, the choice

$$
\mathbb{W}=\frac{\mathbb{D}_{4}^{3}}{\left\langle x_{1}^{2}, x_{2}^{2}, x_{3}^{2}, x_{4}^{2}, x_{3} x_{2}, x_{3} x_{4}, x_{3} x_{1},\right\rangle}
$$

works similarly to the classical calculations over the dual numbers, but the interferences of two different rotation errors are not neglected, that is, $x_{i} x_{j} \neq 0$ for $i \neq j$.

\section{Conflict of Interests}

The authors declare that there is no conflict of interests regarding the publication of this paper.

\section{Acknowledgments}

The research was supported by the project NETME CENTRE PLUS (LO1202). The results of the project NETME CENTRE PLUS (LO1202) were cofunded by the Ministry of Education, Youth and Sports within the support programme "National Sustainability Programme I." Publication of the results was financially supported by the project Popularization of BUT $\mathrm{R} \& D$ results and support systematic collaboration with Czech students CZ.1.07/2.3.00/35.0004.

\section{References}

[1] A. K. Srivastava, S. C. Veldhuis, and M. A. Elbestawit, "Modelling geometric and thermal errors in a five-axis cnc machine tool," International Journal of Machine Tools and Manufacture, vol. 35, no. 9, pp. 1321-1337, 1995.

[2] J. A. Soons, F. C. Theuws, and P. H. Schellekens, "Modeling the errors of multi-axis machines: a general methodology," Precision Engineering, vol. 14, no. 1, pp. 5-19, 1992.

[3] E. Díaz-Tena, U. Ugalde, L. N. López de Lacalle, A. de la Iglesia, A. Calleja, and F. J. Campa, "Propagation of assembly errors in multitasking machines by the homogenous matrix method," International Journal of Advanced Manufacturing Technology, vol. 68, no. 1-4, pp. 1-16, 2013.

[4] L. N. López De Lacalle, A. Lamikiz, O. Ocerin, D. Díez, and E. Maidagan, "The Denavit and Hartenberg approach applied to evaluate the consequences in the tool tip position of geometrical errors in five-axis milling centres," International Journal of Advanced Manufacturing Technology, vol. 37, no. 1-2, pp. 122139, 2008.

[5] S. Aguado, D. Samper, J. Santolaria, and J. J. Aguilar, "Identification strategy of error parameter in volumetric error compensation of machine tool based on laser tracker measurements," International Journal of Machine Tools and Manufacture, vol. 53, no. 1, pp. 160-169, 2012.

[6] J.-H. Jung, J.-P. Choi, and S.-J. Lee, "Machining accuracy enhancement by compensating for volumetric errors of a machine tool and on-machine measurement," Journal of Materials Processing Technology, vol. 174, no. 1-3, pp. 56-66, 2006.

[7] K. G. Ahn and D. W. Cho, "Analysis of the volumetric error uncertainty of a three-axis machine tool by beta distribution," International Journal of Machine Tools and Manufacture, vol. 40, no. 15, pp. 2235-2248, 2000.

[8] Q. Cheng, C. Wu, P. Gu, W. Chang, and D. Xuan, "An analysis methodology for stochastic characteristic of volumetric error in multiaxis CNC machine tool," Mathematical Problems in Engineering, vol. 2013, Article ID 863283, 12 pages, 2013.

[9] G. Hermann, "Geometric error correction in coordinate measurement," Acta Polytechnica Hungarica, vol. 4, no. 1, pp. 47-62, 2007.

[10] W. Tian, W. Gao, W. Chang, and Y. Nie, "Error modeling andsensitivity analysis of a five-axis machine tool," Mathematical Problems in Engineering. In press.

[11] N. L. de Lacalle and A. L. Mentxaka, Machine Tools For High PerFormance Machining, Springer, 2009.

[12] M. Kureš, "Weil algebras associated to functors of third order semiholonomic velocities," Mathematical Journal of Okayama University, vol. 56, no. 1, pp. 117-127, 2014. 


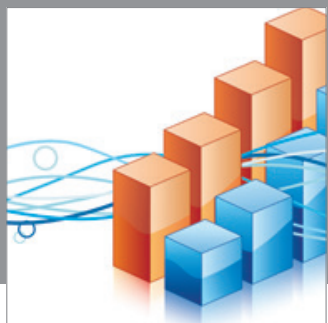

Advances in

Operations Research

mansans

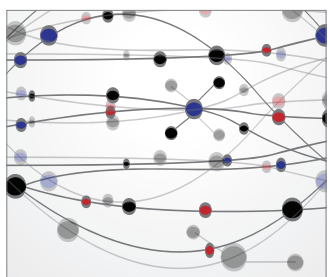

The Scientific World Journal
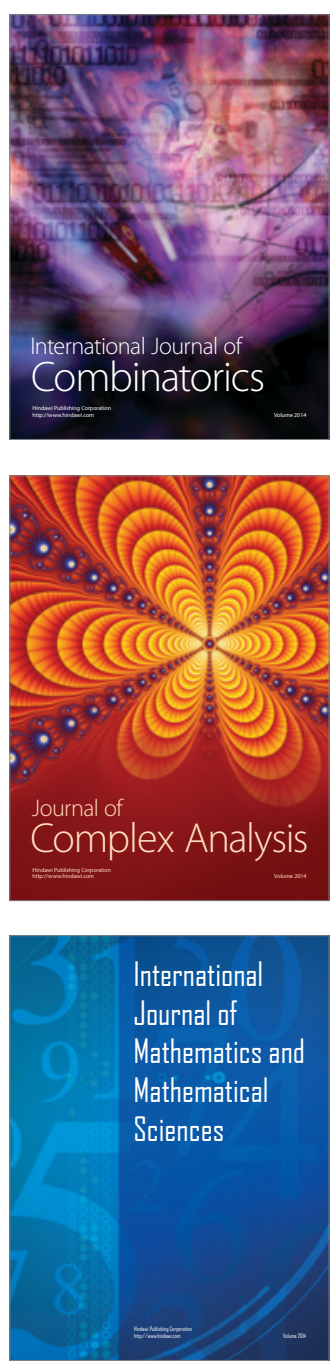
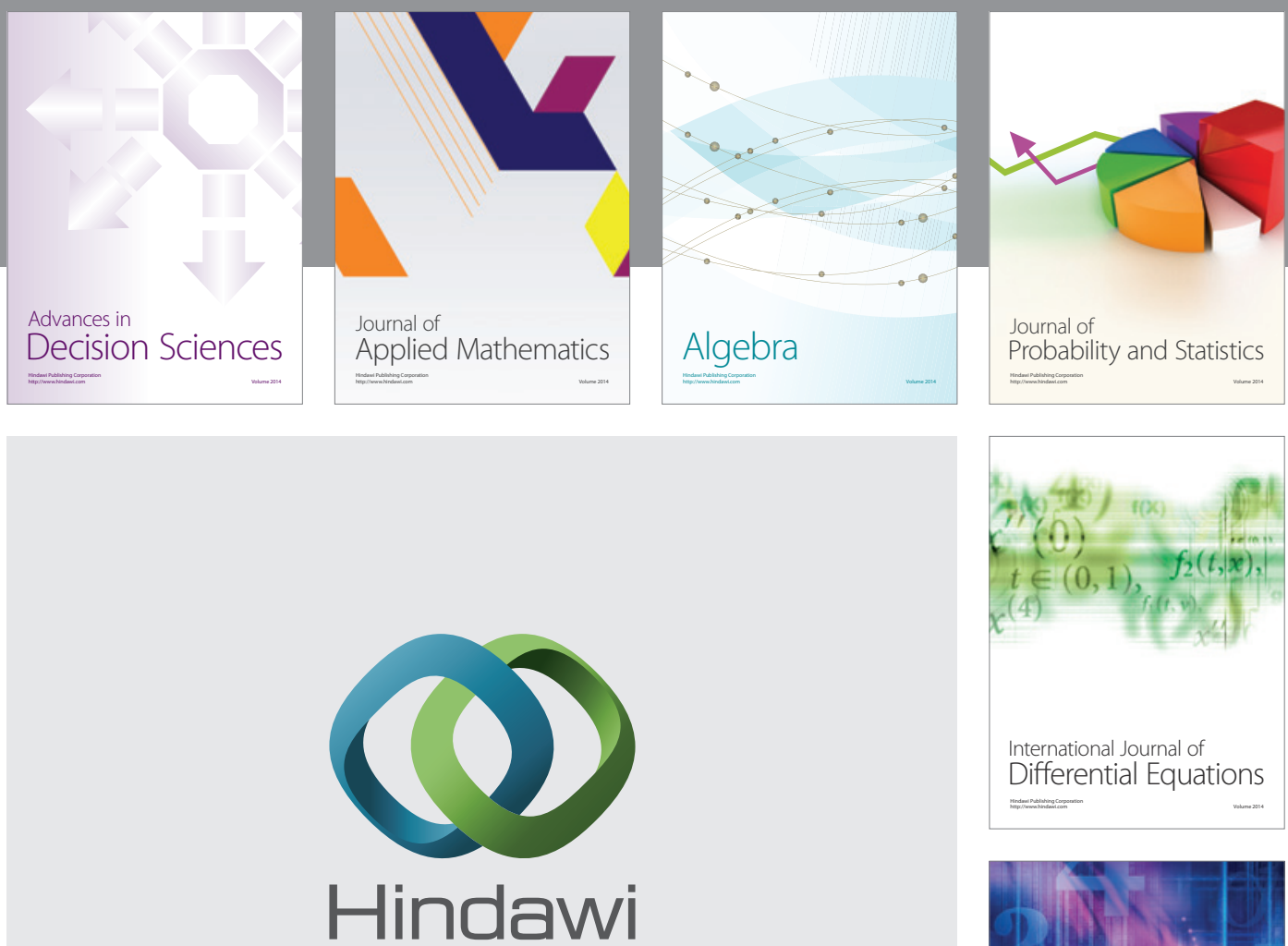

Submit your manuscripts at http://www.hindawi.com
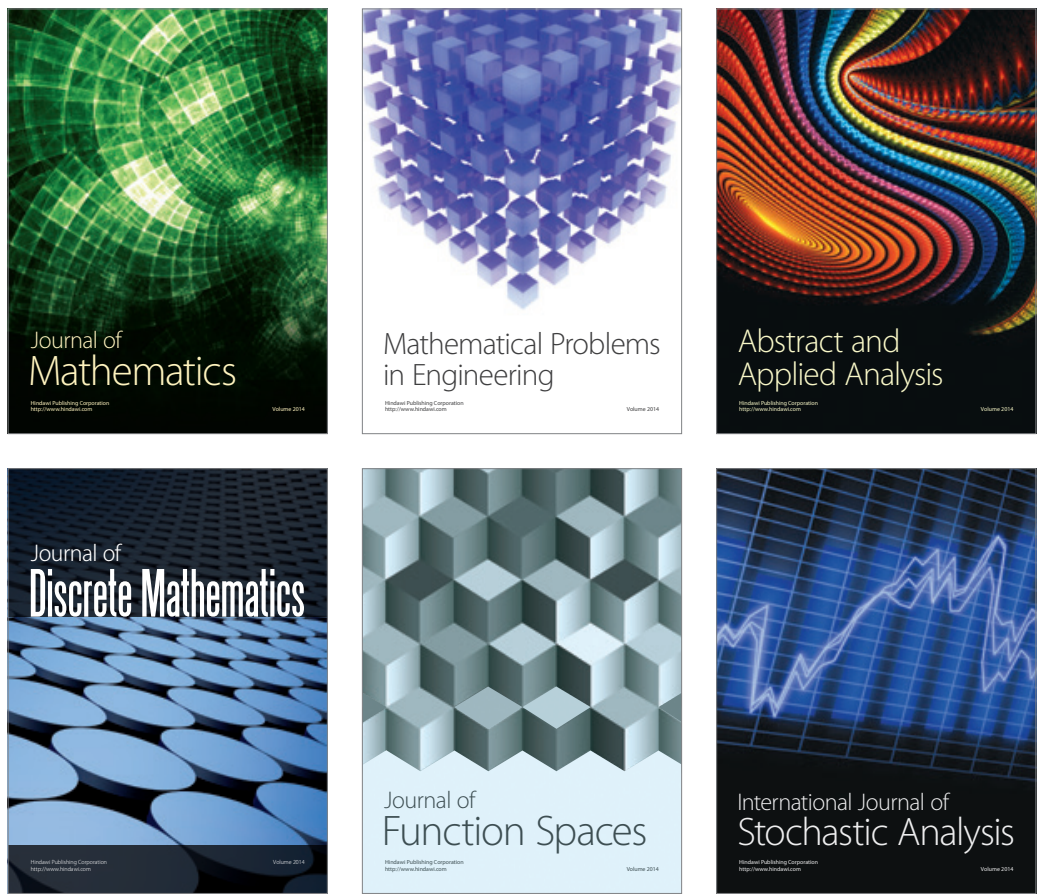

Journal of

Function Spaces

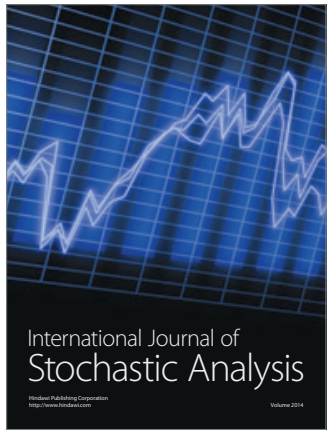

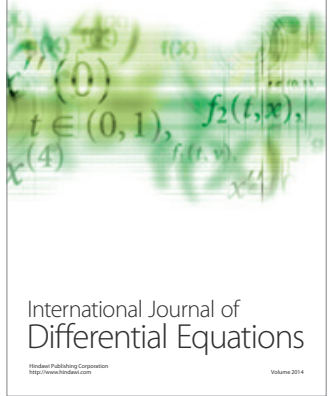
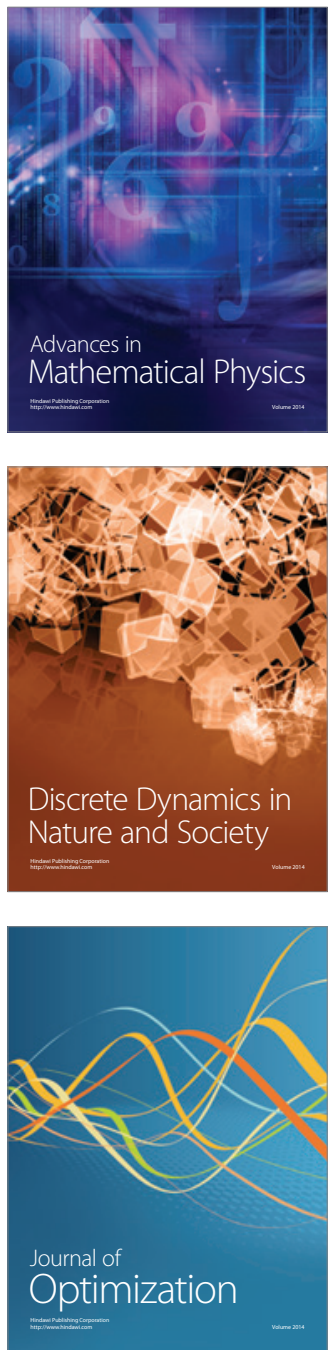